\title{
Anisotropic exciton Stark shift in black phosphorus
}

\author{
A. Chaves,${ }^{1, *}$ Tony Low, ${ }^{2}$ P. Avouris, ${ }^{3}$ D. Çakır,${ }^{4}$ and F. M. Peeters ${ }^{4,1}$ \\ ${ }^{1}$ Universidade Federal do Ceará, Departamento de Física Caixa Postal 6030, 60455-760 Fortaleza, Ceará, Brazil \\ ${ }^{2}$ Electrical and Computer Engineering Department, University of Minnesota, 200 Union Street SE, 4-174 Keller Hall, \\ Minneapolis, Minnesota 55455-0170, USA \\ ${ }^{3}$ IBM Thomas J. Watson Research Center, Yorktown Heights, New York 10598, USA \\ ${ }^{4}$ Department of Physics, University of Antwerp, Groenenborgerlaan 171, B-2020 Antwerp, Belgium \\ (Received 17 November 2014; revised manuscript received 30 March 2015; published 27 April 2015)
}

\begin{abstract}
We calculate the excitonic spectrum of few-layer black phosphorus by direct diagonalization of the effective mass Hamiltonian in the presence of an applied in-plane electric field. The strong attractive interaction between electrons and holes in this system allows one to investigate the Stark effect up to very high ionizing fields, including also the excited states. Our results show that the band anisotropy in black phosphorus becomes evident in the direction-dependent field-induced polarizability of the exciton.
\end{abstract}

DOI: 10.1103/PhysRevB.91.155311

PACS number(s): 78.66.Db, 71.35.-y, 71.70.Ej, 78.20.Jq

\section{INTRODUCTION}

Excitons in semiconductors have been the subject of investigation for many years [1,2]. Such interacting electronhole pair mimics a hydrogen atom, with the hole playing the role of the nucleus [3]. It is well known that the hydrogen atom in the presence of an applied electric field exhibits degeneracy breaking and a quadratic energy shift due to the so-called Stark effect. The experimental observation of such an effect for excitons in bulk semiconductors is, however, hindered by the low electron-hole binding energy - for GaAs, for example, this energy is around $4.8 \mathrm{meV}$ [4], allowing only for very low ionizing electric fields. In such low fields, the exciton binding energy is not significantly modified, albeit the exciton peak broadens due to the decrease of the exciton lifetime. This is a consequence of exciton ionization, which makes the experimental observation of the exciton Stark effect much more challenging. This motivates the study of the Stark effect in quantum wells, which, through the so-called quantum confined Stark effect, can circumvent electron-hole dissociation, allowing the use of higher electric fields [5]. Alternatively, the excitonic Stark effect has been also theoretically investigated in carbon nanotubes [6,7], where the electron-hole binding energies, depending on the nanotube configuration, may reach quite large values [8], allowing for high ionizing fields. Nevertheless, setting up an experiment to detect this effect in carbon nanotubes is a difficult task, which has been achieved only very recently [9]. Strong exciton binding energies are also observed in conjugated polymer chains [10], and inorganic-organic quantum well crystals [11], where exciton Stark shifts for large electric fields have been investigated as well [12-14].

In recent studies on single- or few-layer semiconductors, such as transition metal dichalcogenides and black phosphorus, exciton binding energies are found to be on the order of hundreds of meV [15-18], which brings the possibility of experimentally observing the Stark effect of their excitons. In this context, the case of black phosphorus [19-23], a layered

\footnotetext{
*andrey@fisica.ufc.br
}

material that has recently been fabricated in few-layer form [15] and has a strong potential for technological applications [21,24-28], is of special interest. Its effective mass anisotropy [29-31] leads to an exciton wave function with distinct distributions in different in-plane directions, so that the exciton Stark shift behavior, namely, its electric-field-induced dipole moments and polarizability, is expected to depend on the direction of the applied in-plane electric field.

In this work, we theoretically investigate the excitonic properties of few-layer black phosphorus in the presence of an applied in-plane electric field. The electron-hole pair is described by a continuum model Hamiltonian, where the band anisotropy of black phosphorus is taken into account by assuming anisotropic effective masses, and the influence of the substrate (vacuum) below (above) the phosphorene layer on the dielectric screening is included in the effective electron-hole interaction potential. We propose a finite difference scheme to discretize this Hamiltonian using a nonuniform mesh, which can be adjusted as to take better account of the singularity in the interaction potential. By numerical diagonalization of the discretized Hamiltonian, we obtain a series of exciton energy levels, which is comparable to a modified Rydberg series, adapted to account for the dielectric screening in the material and its surrounding media. The proposed numerical method can be easily adapted for the study of excitons in any layered semiconducting material. Numerical results for the quadratic Stark shift of ground and excited exciton states describe how the effective mass anisotropy of back phosphorus is reflected in its direction-dependent polarizability under external electric fields.

\section{THEORETICAL MODEL}

We consider the exciton Hamiltonian within the effective mass approximation,

$$
H_{\mathrm{exc}}=H_{e}+H_{h}+V\left(\left|\vec{r}_{e}-\vec{r}_{h}\right|\right)
$$

Due to the high anisotropy of black phosphorus energy bands, it is convenient to write the single-particle Hamiltonian in 
Cartesian coordinates $\left(x_{e}, y_{e}, x_{h}, y_{h}\right)$ as

$$
H_{i}=-\frac{\hbar^{2}}{2 m_{i x}} \frac{\partial^{2}}{\partial x_{i}^{2}}-\frac{\hbar^{2}}{2 m_{i y}} \frac{\partial^{2}}{\partial y_{i}^{2}}+q_{i} \vec{F} \cdot \vec{r}_{i},
$$

where $i=e(h)$ represents the electron (hole), $\vec{F}$ is the in-plane applied electric field, $m_{i x(y)}$ is the effective mass of the carrier $i$ in the $x(y)$ direction, and $q_{i}$ is its charge. It is convenient to rewrite the Hamiltonian in units of the Rydberg energy $R_{y}$ and Bohr radius $a_{0}$. We then use relative $(x, y)$ and center-ofmass $(\vec{R})$ coordinates in each direction to simplify the exciton Hamiltonian as

$$
H=-\frac{1}{\mu_{x}} \frac{\partial^{2}}{\partial x^{2}}-\frac{1}{\mu_{y}} \frac{\partial^{2}}{\partial y^{2}}+V\left(\sqrt{x^{2}+y^{2}}\right)+e \vec{F} \cdot \vec{r},
$$

where $\mu_{x(y)}$ is the reduced effective mass in the $x(y)$ direction and the center-of-mass contribution is removed, since the potential does not depend on these coordinates. In order to take proper account of the large dielectric contrast between the vacuum on top of the sample and the substrate below it, the interaction potential $V(r)$, for $r=\sqrt{x^{2}+y^{2}}$, is assumed to be of the Keldysh type [31,32], which in dimensionless form reads

$$
V(r)=-\frac{2 \pi}{\left(\epsilon_{1}+\epsilon_{2}\right) \rho_{0}}\left[H_{0}\left(\frac{r}{\rho_{0}}\right)-Y_{0}\left(\frac{r}{\rho_{0}}\right)\right],
$$

where $H_{0}$ and $Y_{0}$ are Struve and Neumann functions, respectively, $\epsilon_{1(2)}$ is the dielectric constant of the vacuum (substrate) surrounding the phosphorene layer, and $\rho_{0}=D \epsilon /\left(\epsilon_{1}+\epsilon_{2}\right)$ is the screening factor, where $D$ is the effective width of the layer and $\epsilon$ is its dielectric constant, which is isotropic for our problem of interest, as discussed in Ref. [33]. For $n_{l}$ layers of phosphorene on a $\mathrm{SiO}_{2}$ substrate, this factor was found to be $\rho_{0}=n_{l} 10.79 \AA$ [15].

To find the excitonic eigenstates, one can use variational functions as proposed in Refs. [15,17]. However, this kind of approach normally allows one to find only the ground-state energy, or just a few excited states. Hence, in order to obtain several exciton states, we rather numerically diagonalize $H$ within a finite difference scheme, which must be performed with a variable mesh, in order to take better account of the singularity of $V(r)$ as $r \rightarrow 0$. The $x y$ plane is thus discretized in $N_{x} \times N_{y}$ points (here, we take $N_{x}=N_{y}=1020$ ) separated by $h_{j}^{x(y)}=x(y)_{j}-x(y)_{j-1}$, where the mesh varies according to $h_{j}^{x(y)}=\Delta_{\min }^{x(y)}+\Delta_{\max }^{x(y)}\left\{1-\exp \left[-\left(j-N_{x(y)} / 2-1\right) / \delta^{x(y)}\right]\right\}$. This sets the values of $h_{j}^{x(y)}$ for $j>N_{x(y)} / 2$, namely, for $x(y)>0$. The grid is then mirror reflected to the negative part of the $x(y)$ axis. In this way, we can control how close the points are to $r=0$ by adjusting $\Delta_{\text {min }}^{x(y)}$, tune how narrow the mesh is in the neighborhood of this point by adjusting $\delta^{x(y)}$, and set a larger mesh far from $r=0$ by adjusting $\Delta_{\max }^{x(y)}$. Results in this paper are obtained with $\Delta_{\min }^{x(y)}=5 \times 10^{-5} \AA$, $\delta^{x(y)}=100$, and $\Delta_{\max }^{x(y)}=0.4 \AA$.

After discretization, we write the two-dimensional Hamiltonian as a five-diagonals matrix and numerically diagonalize it. The grid points, labeled in the (line, column) form $(i, j)$, are then mapped into a single label $l$. For a nonuniform mesh, diagonalizing this matrix is cumbersome, because the discretized Hamiltonian matrix is asymmetric:

$$
\begin{aligned}
H_{l, l} & =\frac{1}{h_{j+1}^{x} L_{x, j}^{2}}+\frac{1}{h_{j}^{x} L_{x, j}^{2}}+\frac{1}{h_{i+1}^{y} L_{y, i}^{2}}+\frac{1}{h_{i}^{y} L_{y, i}^{2}}+V_{l}, \\
H_{l, l+1} & =-\frac{1}{\mu_{y} h_{i+1}^{y} L_{y, i}^{2}}, \quad H_{l, l-1}=-\frac{1}{\mu_{y} h_{i}^{y} L_{y, i}^{2}}, \\
H_{l, l+N_{y}} & =-\frac{1}{\mu_{x} h_{j+1}^{x} L_{x, j}^{2}}, \quad H_{l, l-N_{y}}=-\frac{1}{\mu_{x} h_{j}^{x} L_{x, j}^{2}}
\end{aligned}
$$

where $L_{x(y), j(i)}^{2}=\left(h_{j(i)+1}^{x(y)}+h_{j(i)}^{x(y)}\right) / 2$. Nevertheless, symmetrization of the Hamiltonian can be achieved by multiplying all the terms in the Hamiltonian by $L_{x, j}^{2} L_{y, i}^{2}$, or equivalently, $\mathcal{B}=\mathcal{L} \mathcal{L} H$, where $\mathcal{L}$ is a diagonal matrix whose diagonal elements are $L_{x, j} L_{y, i}$. Since $\mathcal{L} \mathcal{L} H \mathcal{L}^{-1} \mathcal{L} \psi=E \mathcal{L} \mathcal{L} \psi$, then $\mathcal{B} \mathcal{L}^{-1} \mathcal{L} \psi=E \mathcal{L} \mathcal{L} \psi$ or, equivalently $\mathcal{L}^{-1} \mathcal{B} \mathcal{L}^{-1} \Phi=E \Phi$ (where $\Phi=\mathcal{L} \psi$ ). Hence, diagonalizing $\mathcal{L}^{-1} \mathcal{B} \mathcal{L}^{-1}$, which is symmetric, gives the same eigenenergies as diagonalizing the Hamiltonian itself. This matrix is then numerically diagonalized by Arnoldi's method in order to obtain the exciton states.

\section{RESULTS AND DISCUSSION}

The obtained exciton energies are shown in Fig. 1 for four values of thickness (number of phosphorene layers) of the black phosphorus sample, calculated using the same effective masses as in Ref. [15]. For the sake of comparison, the inset shows (i) the results for $\mathrm{WS}_{2}$, where conduction and valence bands are known to be isotropic with $\mu=\mu_{x}=\mu_{y}=0.16$ [17]; (ii) results for the case where the electron and hole interact via the Coulomb potential, i.e., for the $2 \mathrm{D}$ hydrogen atom, where the permittivity $\epsilon \approx 5.23 \epsilon_{0}$ is adjusted as to fit the ground-state energy of $\mathrm{WS}_{2}$, by means of the analytical expression $\epsilon^{2}=4 R_{y} \mu / E_{0}$, where $E_{0}=0.318 \mathrm{eV}$ is the

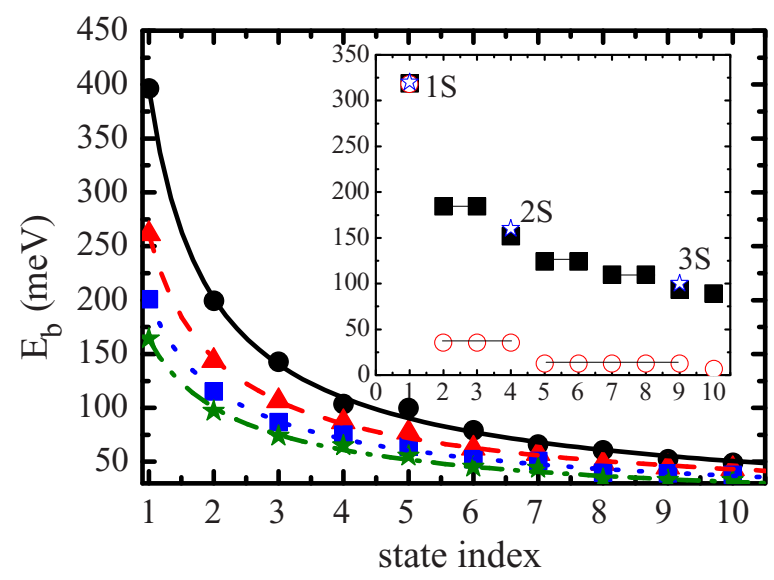

FIG. 1. (Color online) Energies of low-lying exciton states, considering a black phosphorus sample with $n_{l}=1$ (black circles), 2 (red triangles), 3 (blue squares), and 4 (green stars) phosphorene layers. Fitting functions for each case are shown as curves. (Inset) Exciton energy states for $\mathrm{WS}_{2}$ (black squares) and for an electronhole pair interacting by the Coulomb potential (red circles) with an effective relative permittivity $\epsilon_{r}$, along with experimentally obtained values (blue stars, courtesy of the authors of Ref. [16]), for comparison. Degenerate states are connected by horizontal lines to help visualization. 
ground-state exciton binding energy; and (iii) experimental results [16].

Let us first discuss the results in the inset. Notice that the energies obtained from the Coulomb potential are clearly underestimated as compared to those calculated with the Keldysh potential. This is a consequence of the fact that the former diverges faster than the latter as $r \rightarrow 0$. Moreover, results from the Coulomb potential are analytically found to follow $E_{n}=-R_{y} /(n-1 / 2)^{2}$, where the states are $(2 n-1)$ fold degenerate. In fact, this is easily verified with the numerical method proposed in this work. We point out that binding energies calculated with the actual permittivity of $\mathrm{WS}_{2}, \epsilon=13 \epsilon_{0}$, would be even more underestimated, since the effective Rydberg energy is inversely proportional to $\epsilon^{2}$. On the other hand, taking advantage of the fact that the potential in Eq. (4) has a $1 / r$ tail for $r \rightarrow \infty$, one can adjust $\epsilon \approx 1.8 \epsilon_{0}$ as to fit the numerically obtained higher exciton energy states-in this case, due to such smaller effective $\epsilon$, the Coulomb interaction model provides highly overestimated binding energies for the lower states. A twofold degeneracy is still expected due to the circular symmetry of the system, so that positive or negative values of angular momentum $l$ lead to the same energy. However, in the case of the Coulomb potential, additional degeneracies are also observed, due to the $\mathrm{SO}(3)$ symmetry of this potential in two dimensions, which is not the case for the Keldysh potential for $\mathrm{WS}_{2}$. Note that for black phosphorus, not even the twofold degeneracies are observed, since the angular momentum is not a good quantum number in this case, due to the anisotropy of the kinetic energy.

Inspired by the analytical expression for the eigenstates of the Coulomb potential and regarding the fact that the Keldysh potential in Eq. (4) has the Coulomb form as asymptotic behavior, we fit our numerical results by $E_{n}=$ $-\gamma_{1} /\left[\varepsilon(n)^{2}(n-1 / 2)^{2}\right]$, as shown by the curves in Fig. 1 . This expression is equivalent to that for a Coulomb potential, but with an effective permittivity function that depends on the energy level as $\varepsilon(n)=1+\gamma_{2} /(n-1 / 2)^{\gamma_{3}}$. Notice that $\varepsilon(n) \rightarrow 1$ as $n \rightarrow \infty$, so that the fitting function converges to the Rydberg series for high state index $n$. The increase of $\varepsilon(n)$ as $n$ decreases is justified by the fact that lower energy states are more confined in space, so that the contribution of the surrounding medium, where screening is weaker, to the electron-hole interaction is lower, and this situation is equivalent to considering a higher effective permittivity. Fitting parameters are given in Table I. The ground-state exciton energies for $n_{l}=1,2,3$, and 4 phosphorene layers are found to be $-396,-261,-200$, and $-163 \mathrm{meV}$, respectively. This is in good agreement with (namely, less than $10 \%$ lower than) the results obtained by the variational approach [15]. The

TABLE I. Fitting parameters for the exciton spectra in Fig. 1, using the Rydberg-like expression given in the main text, considering a different number of phosphorene layers. The parameter $\gamma_{1}$ is in units of meV, whereas $\gamma_{2}$ and $\gamma_{3}$ are dimensionless.

\begin{tabular}{ccccc}
\hline \hline$n_{l}$ & 1 & 2 & 3 & 4 \\
\hline$\gamma_{1}$ & 2062 & 2958 & 3410 & 2569 \\
$\gamma_{2}$ & 2.094 & 3.416 & 4.32 & 4.031 \\
$\gamma_{3}$ & 0.7665 & 0.7454 & 0.7449 & 0.7783 \\
\hline \hline
\end{tabular}

three lowest energy $s$ states of $\mathrm{WS}_{2}$ are found to be -318.80 , -151.72 , and $-93.56 \mathrm{meV}$, also in good agreement with recent experimental results for this material, which are shown as open blue stars in the inset of Fig. 1 [16], which validates our method.

By using Fermi's golden rule, one can calculate the probability for a photon-induced valence band-to-exciton transition to occur, which is demonstrated to be proportional to $|\langle c|\hat{e} \cdot \vec{p}| v\rangle|^{2}\left|\psi_{n}(0,0)\right|^{2}$, where the first factor is the dipole matrix element between conduction and valence band states. The optically active transition in the case of light polarized in the $x$ direction is between the band states labeled as $\Gamma_{2}^{+}$ and $\Gamma_{4}^{-}$in Ref. [30], which is the one we deal with here. In fact, such a polarization in the lighter effective mass direction has been recently verified experimentally [34]. With this factor set, we just need now to investigate the squared modulus of the exciton envelope function at $r=0$, i.e., $\left|\psi_{n}(0,0)\right|^{2}$, as being the oscillator strength. Indeed, it is reasonable that electron hole recombination processes are more likely when electron and hole are in the same place in real space, i.e., if $\left|\psi_{n}(0,0)\right|^{2}$ is nonzero. Therefore, for isotropic materials such as $\mathrm{WS}_{2}$, the optically active exciton states are only those with $s$-like wave functions. This means that the exciton Rydberg series observed in the experiments performed in Ref. [16] are not the complete exciton energy series: $l \neq 0$ states are missing in the spectrum, and only the states labeled as $1 s, 2 s$, and $3 s$ in the inset of Fig. 1 are observed experimentally in, e.g., Fig. 3 of Ref. [16], with very good quantitative agreement. The $p$ states appear in two-photon experiments, yet with a different energy series, in contrast with the case of Coulomb-like electron-hole interaction, where for each $p$ state there is a degenerate $s$ state. In fact, the $p$ states between the $1 s$ and $2 s$ states observed in our results are consistent with recent experimental observations [35]. In the case of black phosphorus, the angular momentum is no longer a good quantum number, since the kinetic energy operator is not circularly symmetric, but we observe that $n=1$ $\left(E_{1}=-396.37 \mathrm{meV}\right)$ and $n=3\left(E_{3}=-142.78 \mathrm{meV}\right)$ states in the spectrum of Fig. 1 are optically active, i.e., $\left|\psi_{1}(0,0)\right|^{2} \neq 0$ and $\left|\psi_{3}(0,0)\right|^{2} \neq 0$, due to the $s$-like orbital nature of their wave functions (details of the wave functions for these and other exciton states are shown in the Appendix). Thus, we will refer only to these states in the discussion about the exciton Stark effect from here onwards.

Due to the anisotropic band structure of black phosphorus, some physical properties of this material are expected to be direction dependent. Exciton response to applied electric fields, for instance, should be stronger in the direction where the reduced effective mass is lower. This is indeed observed in Fig. 2, which shows, as symbols, the numerically obtained Stark shift for an electric field applied in the $x$ (left panels) and $y$ directions (right panels), considering $n_{l}=1-4$ layers. The quadratic Stark shift follows the expression,

$$
\Delta E=E(F)-E(F=0)=p F+\beta F^{2},
$$

where $F$ is the magnitude of the electric field, $p$ is the intrinsic dipole moment, and $\beta$ is the polarizability parameter. Such a quadratic behavior is, to a good extent, observed in our numerical results, which are well fitted by the quadratic curves in the top panels of Fig. 2, where we assumed $p=0$ and used $\beta$ as a fitting parameter in Eq. (6). The $p=0$ 

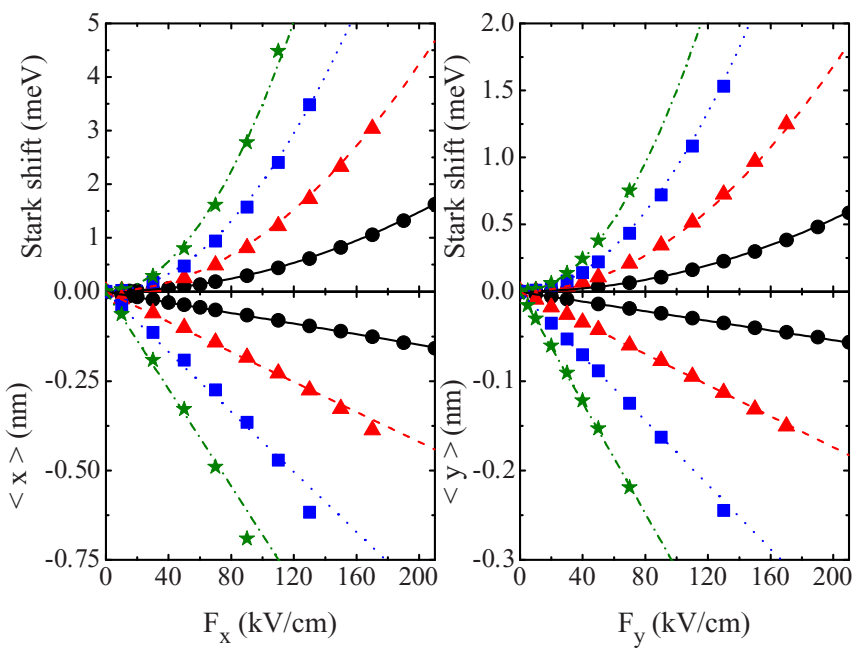

FIG. 2. (Color online) Ground $(n=1)$ state exciton stark shift, considering electric fields applied in the $x$ (left panels) and $y$ directions (right panels), for systems with $n_{l}=1$ (black circles), 2 (red triangles), 3 (blue squares), and 4 (green stars) phosphorene layers. Expectation values of the electron-hole polarization as a function of the applied electric field intensity are shown in the bottom panels. Curves in top and bottom panels are quadratic and linear fittings for the stark shift and polarization, respectively.

assumption is justified by the fact that we are dealing only with nondegenerate states, which do not possess a permanent dipole moment, so that the linear Stark shift can be neglected. The quadratic Stark shift comes from the fact that the applied field is able to induce electron-hole polarization, which depends linearly on the strength of the applied field. Such linear behavior is indeed observed in the bottom panels of Fig. 2, where the numerically obtained polarizations (symbols), i.e., the expectation values of the electron-hole separation $\langle x\rangle$ and $\langle y\rangle$, are well fitted by linear functions (curves). As observed in Fig. 1, the ground-state exciton energy decreases as the number of layers increases. As a consequence, the exciton energy becomes more susceptible to electric field effects for a higher
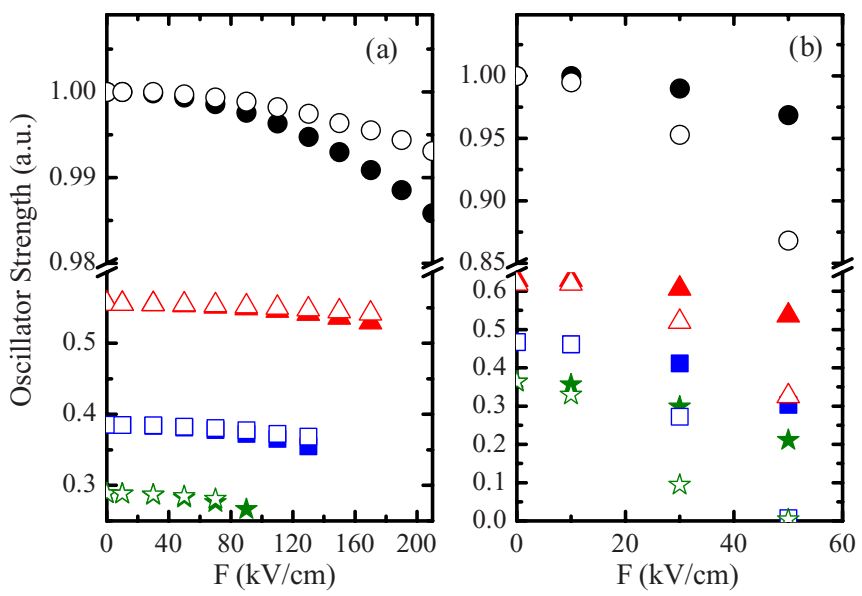

FIG. 3. (Color online) Oscillator strengths for the (a) ground $(n=1)$ and (b) second excited $(n=3)$ exciton states as a function of the strength $F$ of an electric field applied in the $x$ (closed symbols) and $y$ (open symbols) directions. The symbols refer to the different number of layers, as in Fig. 1. number of layers and the Stark shift is more clear in these cases-for instance, considering $n_{l}=4$, the exciton ground state exhibits a Stark shift of $\approx 4.5 \mathrm{meV}$ and a polarization of $\langle x\rangle \approx 25 \AA$ for fields as high as $F=100 \mathrm{kV} / \mathrm{cm}$ applied in the $x$ direction, whereas a much smaller $\Delta E \approx 1.5 \mathrm{meV}$ and $\langle x\rangle \approx 4.5 \AA$ is observed for $n_{l}=1$ in a field twice higher. Nevertheless, even such a small Stark shift is still within the range of energies that are detectable with current experimental techniques (see, e.g., Ref. [36]). It is also clear that electric fields applied in the $x$ direction affect more the exciton polarization and, consequently, lead to higher Stark shift as compared to a field applied in the $y$ direction.

Another interesting feature of the exciton Stark effect in black phosphorus, as compared to the one in conventional bulk semiconductors, is the opportunity to observe such effect also in an excited state. The same study we made for the ground-state exciton in Fig. 2 was also done for the second excited state $(n=3)$, where quite similar features are observed, although small deviations from the quadratic Stark shift are observed at high applied fields, which were previously also found for carbon nanotube exciton Stark shift [6]. A clear difference, however, lies in the stronger Stark shift observed in the $n=3$ case, as compared to the previous case, which is due to its lower binding energy: In the $n_{l}=4$ case, for instance, a $\Delta E \approx 17 \mathrm{meV}$ shift is obtained for an electric field of $50 \mathrm{kV} / \mathrm{cm}$ applied in the $x$ direction. A similar shift is also obtained for $n_{l}=1$, but for a higher field, $F_{x}=110 \mathrm{kV} / \mathrm{cm}$. On the other hand, such lower binding energy also prevents us from investigating the effect up to higher fields without dissociating the electron-hole pair. The polarizabilities of the exciton ground and second excited states, for systems with different number of layers, are summarized in Table II, where the anisotropy of the Stark effect becomes quite evident by the distinct polarizability parameters found for electric fields applied in different directions.

The polarization induced by the field is expected to contribute to a broadening of the excitonic peak in the absorption spectrum and, therefore, hinder the visualization of the Stark effect. Nevertheless, our results for the oscillator strength in Fig. 3 suggest that such contribution is quite small in the ground state, especially for a monolayer: $\left|\psi_{1}(0,0)\right|^{2}$ changes by only $\approx 2 \%(1 \%)$ for fields up to $F_{x(y)}=210 \mathrm{kV} / \mathrm{cm}$, whereas such a change is already observed for a field of $F_{x}=90\left(F_{y}=70\right)$ in the case of four layers. As for the excited state, $\left|\psi_{3}(0,0)\right|^{2}$ changes up to $\approx 14(27) \%$ in the case of $F_{y}=50(30) \mathrm{kV} / \mathrm{cm}$ for $n_{l}=1(4)$, as compared to the zero field case. Results in each panel are divided by the zero field oscillator strength of the $n_{l}=1$ case. This means that the $n_{l}=2,3$, and 4 systems exhibit ground (second excited) state oscillator strengths that are $\approx 55.5 \%$ (61.9\%), 38.5\% (46.7\%), and $28.8 \%$ (36.3\%) of that of the monolayer case, respectively.

TABLE II. Polarizability parameters $\beta_{x}^{n}$ and $\beta_{y}^{n}$ for the $n=1$ and 3 states, in units of $\AA^{2} / \mathrm{mV}$, for electric fields applied in the $x$ and $y$ directions, respectively.

\begin{tabular}{lcccc}
\hline \hline$n_{l}$ & 1 & 2 & 3 & 4 \\
\hline$\beta_{x(y)}^{1}$ & $0.37(0.135)$ & $1.06(0.42)$ & $2.05(0.93)$ & $3.5(1.5)$ \\
$\beta_{x(y)}^{3}$ & $10(4.8)$ & $27(13)$ & $48(23)$ & $65(43)$ \\
\hline \hline
\end{tabular}


Notice that the influence of the electric field on the ground-state oscillator strength is higher when the field is applied in the $x$ direction (closed symbols), whereas for the $n=3$ state, it is higher for $F$ applied in the $y$ direction (open symbols). This can also be understood as a manifestation of the anisotropy of the exciton wave function: Although $n=1$ and 3 are both $s$-like states, we observe that $\left\langle x^{2}\right\rangle>\left\langle y^{2}\right\rangle$ for the former, whereas $\left\langle x^{2}\right\rangle<\left\langle y^{2}\right\rangle$ for the latter (cf. Appendix), which explains such opposite behavior of the oscillator strengths of these states under applied fields.

So far, we have only discussed the exciton energies and anisotropic Stark effect in the specific case of black phosphorus. It is, however, as important to investigate, in general, how the exciton Stark shift parameters in Eq. (6) behave as the effective mass anisotropy $\mu_{y} / \mu_{x}=\alpha$ increases. By defining an effective Bohr radius $a_{0}^{\text {eff }}=a_{0}\left(\epsilon_{1}+\epsilon_{2}\right) / \mu_{x}$ and an effective Rydberg energy $R_{y}^{\text {eff }}=R_{y} \mu_{x} /\left(\epsilon_{1}+\epsilon_{2}\right)$, the dimensionless Hamiltonian Eq. (3) is re-written as

$$
\begin{aligned}
H= & -\frac{\partial^{2}}{\partial x^{2}}-\frac{1}{\alpha} \frac{\partial^{2}}{\partial y^{2}}-\frac{2 \pi}{\rho_{0}}\left[H_{0}\left(\frac{r}{\rho_{0}}\right)-Y_{0}\left(\frac{r}{\rho_{0}}\right)\right], \\
& +e \vec{F}^{\prime} \cdot \vec{r},
\end{aligned}
$$

where $\vec{F}^{\prime}=\left(a_{0}^{\text {eff }} / R_{y}^{\text {eff }}\right) \vec{F}$. The exciton binding energies, obtained by numerical diagonalization of this Hamiltonian, are shown in Fig. 4(a) as a function of the anisotropy parameter $\alpha$. Notice that the effective mass anisotropy lifts the degeneracy of the $p_{x}$ and $p_{y}$ states, so that the $2 s$ state becomes more

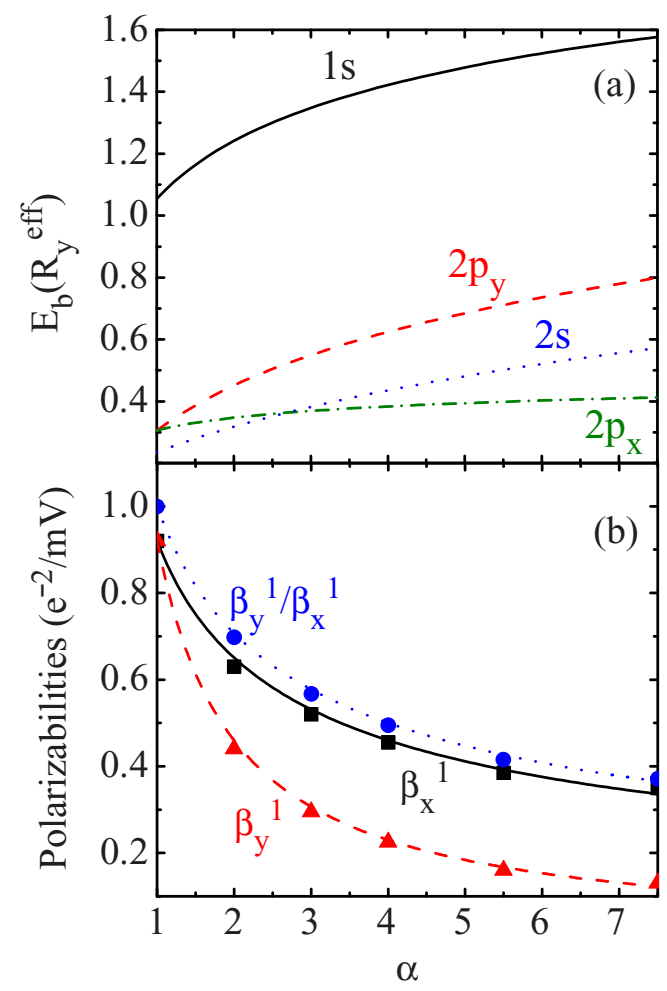

FIG. 4. (Color online) (a) Exciton binding energies as a function of the effective mass anisotropy parameter $\alpha=\mu_{y} / \mu_{x}$. (b) Numerically obtained (symbols) ground-state polarizabilities $\beta_{x(y)}^{1}$ in the $x(y)$ direction, along with their ratio, as a function of the mass anisotropy parameter. Numerical results for $\beta_{x}^{1}, \beta_{y}^{1}$ and $\beta_{y}^{1} / \beta_{x}^{1}$ are fit to $0.92 \alpha^{-0.5}$ (solid), $0.92 \alpha^{-1}$ (dashed), and $\alpha^{-0.5}$ (dotted), respectively. energetic than the $2 p_{x}$ state for $\alpha \gtrsim 2.6$, as verified by the exciton wave functions for monolayer black phosphorus (see Appendix), where $\alpha \approx 7.3$.

The quadratic Stark shift Eq. (6) is the second-order correction to the energy levels, within perturbation theory, for an electron-hole pair in the presence of an external electric field. Regardless the anisotropy of the effective masses, the $x(y)$ component of the intrinsic dipole moment $p_{x(y)}=$ $e\langle\psi|x(y)| \psi\rangle$ in this expression is zero for $s$-like states, which is clear from the parity of their wave functions. In the case of monolayer black phosphorus, these are the $n=1$ and 3 states. The polarizability of the $n$th state is given by

$$
\beta_{x(y)}^{n}=e^{2} \sum_{m \neq n} \frac{\left|\left\langle\psi_{m}|x(y)| \psi_{n}\right\rangle\right|^{2}}{E_{m}-E_{n}} .
$$

It is clear that the polarizability will depend on the spatial distribution of the wave functions, which is anisotropic in the case of anisotropic effective masses investigated here. However, since we do not have analytical expressions for the exciton wave functions $\psi_{n}(x, y)$ in Eq. (8), this quantity is more conveniently obtained by parabolic fitting of our numerical results. The numerically obtained polarizabilities $\beta_{x}^{1}, \beta_{y}^{1}$, along with their ratio $\beta_{y}^{1} / \beta_{x}^{1}$, are shown as symbols in Fig. 4(b). They are demonstrated to decay approximately as $0.92 \alpha^{-0.5}$ (solid), $0.92 \alpha^{-1}$ (dashed), and $\alpha^{-0.5}$ (dotted), respectively, as the effective mass anisotropy increases.

\section{CONCLUSIONS}

We have calculated the excitonic states in few-layer black phosphorus in the presence of an external electric field by numerical diagonalization of the effective mass Hamiltonian. The method developed here is shown to be easily adapted for other layered materials and agrees well with recent experiments on $\mathrm{WS}_{2}$. Due to the non-Coulomb effective interaction potential between electron and hole in such 2D systems, the degeneracy coming from the $\mathrm{SO}(3)$ symmetry of the planar hydrogen atom model is lifted, whereas the band anisotropy is responsible for lifting the degeneracy of angular momentum eigenstates. Even so, the exciton spectrum in black phosphorus can still be satisfactorily fitted by a hydrogenlike expression. Due to the high binding energies, excitons can withstand strong in-plane electric fields without dissociating [37]. This allowed us to observe Stark shifts up to $\approx 15 \mathrm{meV}$, with electric fields up to $\approx 200 \mathrm{kV} / \mathrm{cm}$, not only for the ground-state exciton, but also for excited states, without significant depreciation of the oscillator strength. We believe that the clear and unusual anisotropic exciton Stark shift predicted here may stimulate further photoluminescence and reflectance experiments in few-layer black phosphorus under in-plane electric fields in the near future.

\section{ACKNOWLEDGMENTS}

Discussions with J. M. Pereira Jr. and J. S. de Souza are gratefully acknowledged. This work was supported by the Brazilian Council for Research $(\mathrm{CNPq})$ through the PQ and Science Without Borders programs, the Flemish Science Foundation (FWO-Vl), the Methusalem programme of the Flemish government, and the Bilateral program (CNPq-FWO) between Flanders and Brazil. 


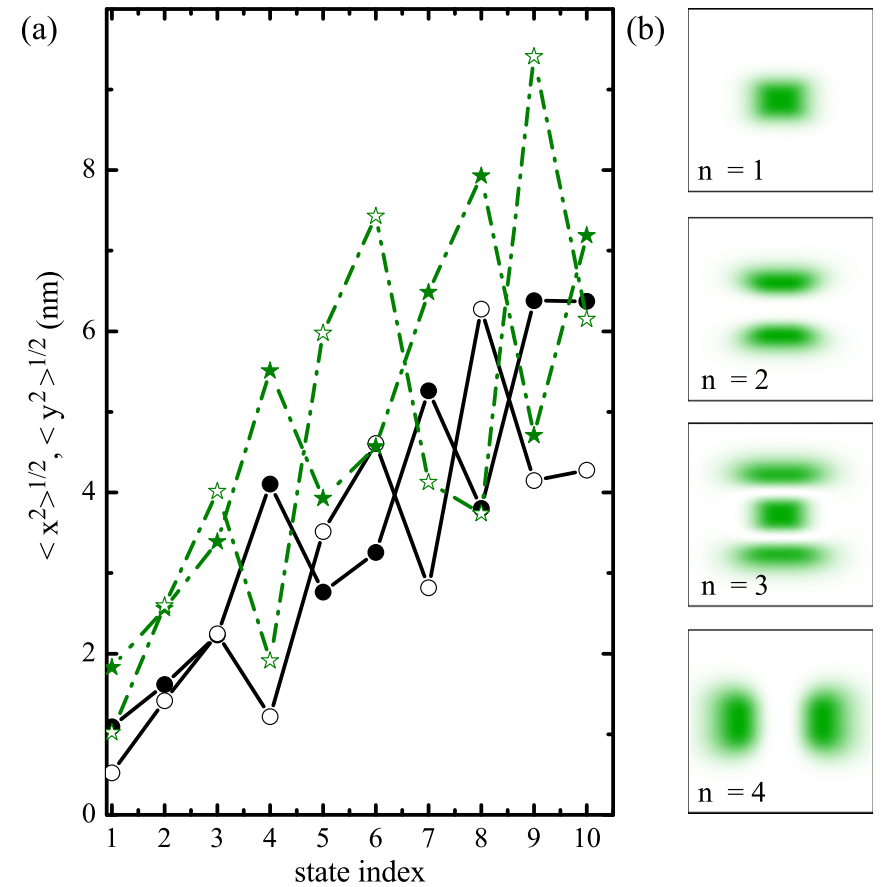

FIG. 5. (Color online) (a) Average width of the exciton function in each direction for $n_{l}=1$ (black circles) and 4 (green stars). Open (full) symbols are results for $\left\langle y^{2}\right\rangle\left(\left\langle x^{2}\right\rangle\right)$. (b) Wave functions for the four low-lying exciton states, considering $n_{l}=4$ black phosphorus in the absence of electric fields.

\section{APPENDIX : EXCITON WAVE FUNCTIONS}

Let us discuss some properties of the wave functions of the exciton states shown in Fig. 1. Figure 5(a) shows the width of each exciton state wave function, considering $n_{l}=1$ (black circles) and 4 (green stars). Lines connecting the symbols curves are shown just as a guide to the eye. The anisotropy [namely, the difference between $\left\langle x^{2}\right\rangle$ (open symbols) and $\left\langle y^{2}\right\rangle$ (full symbols)] is more evident for some specific exciton states. As verified by the wave functions for the four low-lying exciton energy states for the $n_{l}=4$ case, shown in Fig. 1(b), the $n=$ 1 state resembles a $1 s$ orbital, as expected, but its width is twice larger in the direction of lighter effective mass, namely, the $x$ direction. Subsequent states resemble $2 p_{y}, 2 s$, and $2 p_{x}$ orbitals, respectively. Similar wave functions are also found in Fig. 7 of Ref. [31] for $n_{l}=1$. The $n=2$ state exhibits $\left\langle x^{2}\right\rangle \sim\left\langle y^{2}\right\rangle$, which is unusual for a $p_{y}$ state, that is normally more spread in the $y$ direction. However, this is compensated in the case of phosphorene by its lighter effective mass in the $x$ direction. The $n=3$ state, which is a $2 s$-like orbital, exhibits a slightly larger width in the $y$ direction, which is more evident in the $n_{l}=4$ case, as compared to $n_{l}=1$. This has consequences for its oscillator strength, as discussed in the main manuscript. From the widths shown in Fig. 1(a), one can infer that the exciton wave function in both directions is spread over tens of angstrons, i.e., over a region many times the interatomic distance $a \approx 2 \AA$ in black phosphorus [15], as required for the validity of the Wannier-Mott theory of excitons used here. In fact, just like in carbon nanotubes [38,39], excitons in fewlayer black phosphorus undergo low screening outside the layer, resulting in quite large electron-hole binding energies, as opposed to those of Frenkel excitons. Nevertheless, the dielectric function in the layer itself is of the same order of magnitude of those of other semiconductor materials [15], such as $\mathrm{Si}$ and GaAs (namely, $\epsilon \approx 10 \epsilon_{0}$ ), which leads to the large spatial extension of the exciton wave function observed here, thus justifying the use of Wannier-Mott theory.
[1] R. J. Elliot, Phys. Rev. 108, 1384 (1957).

[2] V. D. Kulakovskii, V. G. Lysenk, and V. B. Timofeev, Sov. Phys. Usp. 28, 735 (1985).

[3] S. W. Koch, M. Kira, G. Khitrova, and H. M. Gibbs, Nature Materials 5, 523 (2006).

[4] A. Venu Gopal, R. Kumar, A. S. Vengurlekar, A. Bosacchi, S. Franchi, and L. N. Pfeiffer, J. Appl. Phys. 87, 1858 (2000).

[5] D. A. B. Miller, D. S. Chemla, T. C. Damen, A. C. Gossard, W. Wiegmann, T. H. Wood, and C. A. Burrus, Phys. Rev. Lett. 53, 2173 (1984).

[6] V. Perebeinos, J. Tersoff, and P. Avouris, Phys. Rev. Lett. 92, 257402 (2004).

[7] V. Perebeinos and P. Avouris, Nano Lett. 7, 609 (2007).

[8] T. Ando, J. Phys. Soc. Jpn. 66, 1066 (1997).

[9] M. Yoshida, Y. Kumamoto, A. Ishii, A. Yokoyama, and Y. K. Kato, Appl. Phys. Lett. 105, 161104 (2014).

[10] L. Sebastian and G. Weiser, Phys. Rev. Lett. 46, 1156 (1981).

[11] K. Tanaka, T. Takahashi, T. Kondo, T. Umebayashi, K. Asai, and K. Ema, Phys. Rev. B 71, 045312 (2005).

[12] K. Tanaka and T. Kondo, Sci. Technol. Adv. Mater. 4, 599 (2003).

[13] G. Weiser, Phys. Rev. B 45, 14076 (1992).
[14] G. Weiser, L. Legrand, T. Barisien, A. A. Choueiry, M. Schott, and S. Dutremez, Phys. Rev. B 81, 125209 (2010).

[15] A. Castellanos-Gomez, L. Vicarelli, E. Prada, J. O. Island, K. L. Narasimha-Acharya, S. I. Blanter, D. J. Groenendijk, M. Buscema, G. A. Steele, J. V. Alvarez, H. W. Zandbergen, J. J. Palacios, and H. S. J. van der Zant, 2D Materials 1, 025001 (2014).

[16] A. Chernikov, T. C. Berkelbach, H. M. Hill, A. Rigosi, Y. Li, O. B. Aslan, D. R. Reichman, M. S. Hybertsen, and T. F. Heinz, Phys. Rev. Lett. 113, 076802 (2014).

[17] T. C. Berkelbach, M. S. Hybertsen, and D. R. Reichman, Phys. Rev. B 88, 045318 (2013).

[18] K. Fai Mak, K. He, C. Lee, G. Hyoung Lee, J. Hone, T. F. Heinz, and J. Shan, Nature Materials 12, 207 (2013).

[19] H. Liu, Y. Du, Y. Deng, and P. D. Ye, Chem. Soc. Rev. (2015).

[20] H. Liu, A. T. Neal, Z. Zhu, Z. Luo, X. Xu, D. Tománek, and Peide D. Ye, ACS Nano 8, 4033 (2014).

[21] L. Li, Yijun Yu, G. J. Ye, Q. Ge, X. Ou, H. Wu, D. Feng, X. H. Chen, and Y. Zhang, Nature Nanotechnology 9, 372 (2014).

[22] S. P. Koenig, R. A. Doganov, H. Schmidt, A. H. Castro Neto, and B. Özyilmaz, Appl. Phys. Lett. 104, 103106 (2014).

[23] D. Çakır, H. Sahin, and F. M. Peeters, Phys. Rev. B 90, 205421 (2014). 
[24] M. Engel, M. Steiner, and P. Avouris, Nano Lett. 14, 6414 (2014).

[25] M. Buscema, D. J. Groenendijk, S. I. Blanter, G. A. Steele, H. S. J. van der Zant, and A. Castellanos-Gomez, Nano Lett. 14, 3347 (2014).

[26] H. Yuan, X. Liu, F. Afshinmanesh, W. Li, G. Xu, J. Sun, B. Lian, G. Ye, Y. Hikita, Z. Shen, S.-C. Zhang, X. Chen, M. Brongersma, H. Y. Hwang, and Y. Cui, arXiv:1409.4729.

[27] T. Low, M. Engel, M. Steiner, and P. Avouris, Phys. Rev. B 90, 081408 (2014).

[28] A. Morita, Appl. Phys. A 39, 277 (1986).

[29] J. Qiao, X. Kong, Z.-X. Hu, F. Yang, and W. Ji, Nature Communications 5, 4475 (2014).

[30] P. Li and I. Appelbaum, Phys. Rev. B 90, 115439 (2014).

[31] A. S. Rodin, A. Carvalho, and A. H. Castro Neto, Phys. Rev. B 90, 075429 (2014).

[32] L. V. Keldysh, Pis'ma Zh. Eksp. Teor. Fiz. 29, 716 (1979) [JETP Lett. 29, 658 (1980)].

[33] T. Low, R. Roldán, H. Wang, F. Xia, P. Avouris, L. M. Moreno, and F. Guinea, Phys. Rev. Lett. 113, 106802 (2014).
[34] X. Wang, A. M. Jones, K. L. Seyler, V. Tran, Y. Jia, H. Zhao, H. Wang, L. Yang, X. Xu, and F. Xia, arXiv:1411.1695.

[35] Z. Ye, T. Cao, K. O. Brien, H. Zhu, X. Yin, Y. Wang, S. G. Louie, and X. Zhang, Nature (London) 513, 214 (2014).

[36] B. D. Gerardot, S. Seidl, P. A. Dalgarno, R. J. Warburton, D. Granados, J. M. Garcia, K. Kowalik, O. Krebs, K. Karrai, A. Badolato, and P. M. Petroff, Appl. Phys. Lett. 90, 041101 (2007).

[37] Since the electric field potential diverges to $-\infty$ as $r \rightarrow \infty$, any electron-hole pair is expected to eventually dissociate. However, such dissociation is highly unlikely to occur before the exciton decays, since it would require tunneling through a significant thick potential barrier, provided by the strong electron-hole interaction potential.

[38] D. T. Nguyen, C. Voisin, Ph. Roussignol, C. Roquelet, J. S. Lauret, and G. Cassabois, Phys. Rev. Lett. 107, 127401 (2011).

[39] P. Avouris, M. Freitag, and V. Perebeinos, Nature Photonics 2, 341 (2008). 